\title{
On the Variability of Supergiant Stars
}

\author{
G. Burki and F. Kienzle \\ Geneva Observatory, 1290 Sauverny, Switzerland
}

\section{Introduction}

The Ia-type supergiants of spectral type $B$ to $G$ are probably all variable in radial velocity and luminosity; in addition, on the average, the amplitude of the variations increases with increasing intrinsic luminosity and are larger for the earliest and latest spectral types (Abt, 1957; Maeder \& Rufener, 1972; Burki et al., 1978; Grenon, 1993). The variations are not strictly periodic, but can be frequently described by a characteristic time $T_{c}$, i.e., some kind of 'most probable period' (e.g., Sterken, 1977; Rufener et al., 1978; Percy et al., 1979; van Genderen et al., 1992).

It is possible to define a $T_{\mathrm{c}}$-luminosity-colour relation for the supergiant stars (Maeder \& Rufener, 1972; Burki, 1978). However, this relation exhibits a large dispersion, due to the imprecise luminosity and colour determinations for these stars and, essentially, to the fact that each supergiant can show very different $T_{c}$ values. Good determinations of $T_{c}$ require very long-term, continuous, photometric monitorings.

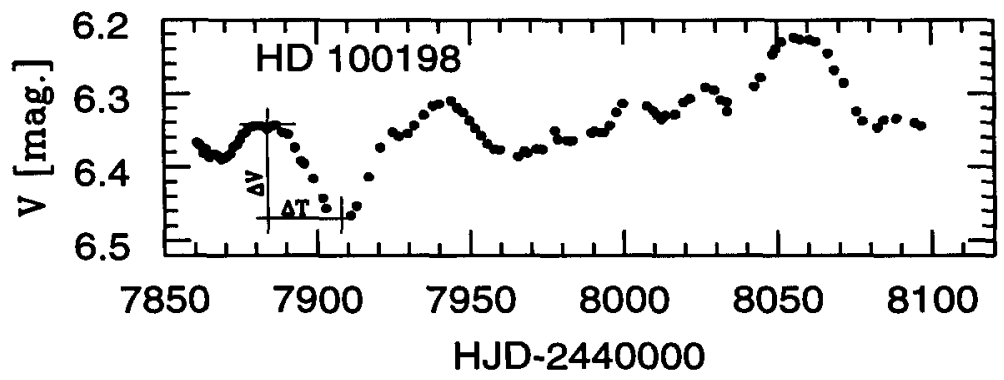

Figure 1. A portion of the photometric observations on HD 100198

\section{A new description of the supergiant variability}

A small group of bright, circumpolar, southern supergiant stars has been selected for a continuous monitoring from the Geneva station at the E.S.O. La Silla Observatory. A first result of this monitoring was the discovery that V810 Centauri (G0Ia) is a double-mode Cepheid-like pulsator, in addition to its supergiant behaviour (Burki, 1994). We present here the results of the preliminary analysis on HD 93737 (A0Ia/ab), HD 100198 (A3Ia) and HD 100261 (F7Ia/ab). It is 
clearly apparent in Figure 1 that, taking into account the high-precision (better than 5 millimag.) and the very good temporal coverage of the data, very faint variations can be described. These data allow us to adopt a new description of the supergiant variability : on the light curve, each increasing or decreasing portion is characterized by $\Delta V$, the luminosity variation, and by $\Delta T$, the duration of this variation. Note that this method was already used by Burki et al. (1982) to describe the variability of the extreme supergiant $\zeta^{1}$ Sco. In Figure 2 are plotted the data $\Delta V$ vs. $\Delta T$. On the basis of this small sample, we can tentatively postulate that the slope $\Delta V / \Delta T$ decreases from $B$ to $G$ spectral type. In addition, at a given spectral type, the maximum value of $\Delta V$ (and, thus, also of $\Delta T$ ) increases with increasing intrinsic luminosity.

A more complete analysis, based on a larger sample of supergiants observed by us in the Geneva photometric system or by various other authors, is in progress.

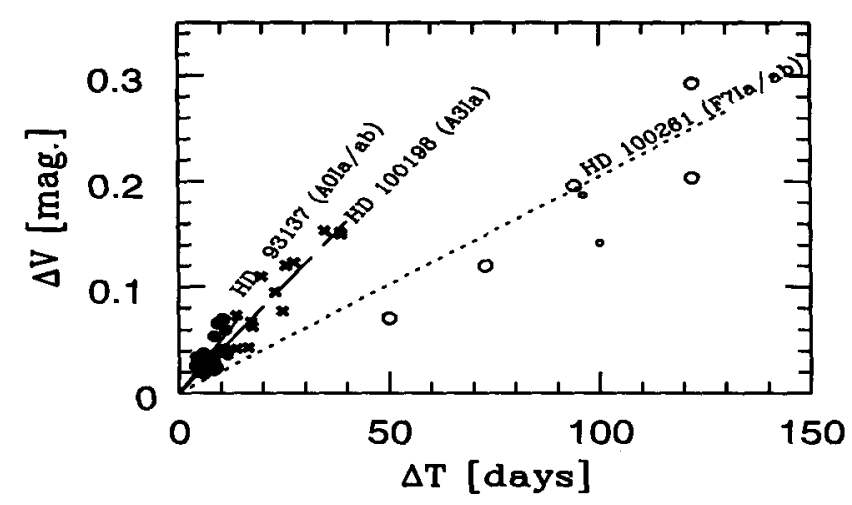

Figure 2. Relation between the luminosity variations $\Delta V$ and the corresponding time intervals $\Delta T$ for three supergiants

\section{References}

Abt, H.A. 1957, ApJ, 126, 138

Burki, G. 1978, A\&A, 65, 357

Burki, G. 1994, in: The Impact of Long-Term Monitoring on Variable Star Research, Eds. C. Sterken \& M. de Groot, Kluwer, Dordrecht, 247

Burki, G., Maeder, A., \& Rufener, F. 1978, A\&A, 65, 363

Grenon, M. 1993, in Inside the Stars, Eds. W.W. Weiss \& A. Baglin, ASP Conf. Series, 40,693

Maeder, A., \& Rufener, F. 1972, A\&A, 20, 437

Percy, J.R., Barkerville, I., \& Trevorrow, W. 1979, PASP, 91, 368

Rufener, F., Maeder, A., \& Burki, G. 1978, A\&AS, 31, 179

Sterken, C. 1977, A\&A, 57, 361

van Genderen, A.M., et al. 1992, A\&A, 258, 316 